\title{
Current and future immunotherapy approaches in ovarian cancer
}

\author{
Nazım Can Demircan ${ }^{1 \wedge}$, Stergios Boussios ${ }^{2,3^{\wedge}}$, Tolga Tasci $^{4}$, Mehmet Akif Öztürk $^{{ }^{\wedge}}$ \\ ${ }^{1}$ Division of Medical Oncology, Department of Internal Medicine, Marmara University Faculty of Medicine, Istanbul, Turkey; ${ }^{2}$ Department of \\ Medical Oncology, Medway NHS Foundation Trust, Gillingham, Kent, UK; ${ }^{3}$ AELIA Organization, 9th Km Thessaloniki - Thermi, Thessaloniki, \\ Greece; ${ }^{4}$ Department of Obstetrics \& Gynecology, ${ }^{5}$ Division of Medical Oncology, Department of Internal Medicine, Bahcesehir University Faculty \\ of Medicine, Istanbul, Turkey \\ Contributions: (I) Conception and design: All authors; (II) Administrative support: None; (III) Provision of study materials or patients: None; (IV) \\ Collection and assembly of data: All authors; (V) Data analysis and interpretation: All authors; (VI) Manuscript writing: All authors; (VII) Final \\ approval of manuscript: All authors. \\ Correspondence to: Nazım Can Demircan. Division of Medical Oncology, Marmara University Faculty of Medicine, Istanbul, Turkey. \\ Email: ncdemircan@gmail.com.
}

\begin{abstract}
Ovarian cancer (OC) is the major cause of gynecologic cancer deaths and relapse is common despite advances in surgery and systemic chemotherapy. Therefore, novel treatments are required to improve long-term outcomes of the disease. Efficacy of immunotherapy was demonstrated in many tumors and it has been since incorporated into clinical practice for them. Although early data form preclinical studies imply that OC has an immunogenic microenvironment, immune checkpoint inhibitors (ICIs) did not produce favorable results in clinical trials to date. This review will highlight data from clinical studies regarding immunotherapy in OC and its combination with other agents as well as immunologic prospects which could strengthen the therapeutic armament against the disease in the future.
\end{abstract}

Keywords: Immune checkpoint inhibitors (ICIs); immunotherapy; ovarian cancer (OC)

Submitted Apr 08, 2020. Accepted for publication Jun 10, 2020.

doi: 10.21037/atm-20-4499

View this article at: http://dx.doi.org/10.21037/atm-20-4499

\section{Introduction}

Ovarian cancer (OC) is the second most common gynecologic cancer in developed countries and the leading cause of gynecologic cancer mortality $(1,2)$. Epithelial ovarian cancer (EOC) accounts for the majority of the disease which is primarily treated with debulking surgery and neoadjuvant or adjuvant platinum-based chemotherapy. Despite this approach, most patients eventually experience relapse and receive systemic chemotherapy depending on platinum sensitivity.

Evidence from studies in the last decades suggest that OC may be an immunogenic tumor. Zhang et al. demonstrated that presence of intratumoral CD3 $+\mathrm{T}$ cells was associated with longer survival in advanced OC while Schlienger et al. reported anti-tumor immune response in the form of interferon- $\gamma$ (IFN- $\gamma$ ) secretion by $\mathrm{T}$ cells in peripheral blood or ascites of OC patients $(3,4)$. Although restoration of immunity against $\mathrm{OC}$ seems rational on this basis, early studies investigating the strategy using cytokines yielded conflicting results. IFN- $\gamma$, an anti-viral protein known to enhance antigen presentation to $\mathrm{T}$ cells, had modest efficacy when administered intraperitoneally in OC patients and negative impact on survival when its subcutaneous form was added to carboplatin/paclitaxel regimen in first-line treatment of advanced OC $(5,6)$. Interleukins (ILs) are other immunostimulatory cytokines,

\footnotetext{
${ }^{\wedge}$ Nazim Can Demircan, ORCID: 0000-0001-6630-5278; Stergios Boussios, ORCID: 0000-0002-2512-6131; Mehmet Akif Öztürk, ORCID: 0000$0003-1365-7944$.
} 
Table 1 Immune checkpoint inhibitor monotherapy trials in ovarian cancer

\begin{tabular}{|c|c|c|c|c|c|}
\hline Reference & Study characteristics & Treatment & ORR (\%) & mPFS (months) & $\begin{array}{c}\text { mOS } \\
\text { (months) }\end{array}$ \\
\hline Hamanishi et al. (15) & $\begin{array}{l}\text { Phase II, PTR-EOC; } n=20, \geq 2 \text { lines of } \\
\text { CT }(100 \%)\end{array}$ & Nivolumab 1 or $3 \mathrm{mg} / \mathrm{kg}$ q2w & 15 & 3.5 & 20 \\
\hline Liu et al. (16) & $\begin{array}{l}\text { Phase } \mathrm{Ib}, E O C, n=12 ; \geq 2 \text { lines of CT } \\
(92 \%)\end{array}$ & Atezolizumab 15 mg/kg q3w & 22.2 & 2.9 & 11.3 \\
\hline $\begin{array}{l}\text { Varga et al. (18) } \\
\text { (KEYNOTE-028) }\end{array}$ & $\begin{array}{l}\text { Phase Ib, EOC; } n=26, \geq 2 \text { lines of CT } \\
(88 \%)\end{array}$ & $\begin{array}{l}\text { Pembrolizumab } 10 \text { mg/kg } \\
\text { q3w }\end{array}$ & 11.5 & 1.9 & 13.8 \\
\hline $\begin{array}{l}\text { Matulonis et al. (19) } \\
\text { (KEYNOTE-100) }\end{array}$ & $\begin{array}{l}\text { Phase II, EOC; Cohort A ( } n=285): 1-3 \\
\text { prior lines, PFI =3-12 months }\end{array}$ & Pembrolizumab 200 mg q3w & A: 7.4 & 2.1 for both & A: NR \\
\hline NCT01611558 (21) & $\begin{array}{l}\text { Phase II, recurrent PTS-OC, } n=40,1-4 \\
\text { prior lines of CT }\end{array}$ & $\begin{array}{l}\text { Ipilimumab } 10 \mathrm{mg} / \mathrm{kg} \text { q3w } \times 4 \\
\text { followed by } 10 \mathrm{mg} / \mathrm{kg} \mathrm{q} 12 \mathrm{w}\end{array}$ & 10.3 & NR & NR \\
\hline
\end{tabular}

CT, chemotherapy; EOC, epithelial ovarian cancer; mOS, median overall survival; mPFS, median progression-free survival; NR, not reported; ORR, objective response rate; PFI, platinum-free interval; PTR-EOC, platinum-resistant epithelial ovarian cancer; PTS-OC, platinum-sensitive ovarian cancer.

among which IL-2 and IL-18 demonstrated some activity in various OC settings but data is limited to phase I or II trials (7-9). Additional prospective research is thus warranted to integrate this type of immunotherapy to OC management.

Immune checkpoint inhibitors (ICIs) are novel agents which exert immunostimulatory effects by antagonizing programmed cell death receptor 1 (PD-1), its ligand PDL1 or cytotoxic T lymphocyte antigen 4 (CTLA-4). ICIs have become an established treatment option in many malignancies like advanced lung cancer and malign melanoma but for OC there is no specific immunotherapeutic agent approved yet. Nevertheless, immune checkpoints may be potential targets for activating anti-tumor immunity in OC. Hamanishi et al. reported high PD-L1 expression in $68 \%$ of tissue samples from 70 OC patients whereas Maine et al. revealed that monocytes derived from peripheral blood and ascites of OC patients had significantly higher PD-L1 expression compared to benign or borderline over tumors (10,11). Among EOC subtypes, high grade serous ovarian cancer (HGSOC) is most common and it showed higher PD-L1 positivity and more CD8+ tumor infiltrating lymphocytes (TILs) than less common histologies $(12,13)$. These findings altogether support the rationale that ICIs may be a promising treatment strategy for EOC and in particular, HGSOC cases.

This review will summarize data from clinical trials evaluating ICIs in OC and also address future aspects of immunologic therapy for the disease.

\section{ICI monotherapy}

Blockade of PD-1 or PD-L1 is one major mechanism by which immunotherapy acts. PD-1 is a cell surface protein that interacts with PD-L1 expressed by tumor cells. This interaction stimulates exhaustion of peripheral effector $\mathrm{T}$ cells and conversion of effector $\mathrm{T}\left(\mathrm{T}_{\text {eff }}\right)$ cells to regulatory $\mathrm{T}\left(\mathrm{T}_{\mathrm{reg}}\right)$ cells, thereby limiting immune response (14). Nivolumab and pembrolizumab are anti-PD-1 monoclonal antibodies while atezolizumab, avelumab and durvalumab inhibit PD-L1.

Single-agent PD-1/PD-L1 inhibitors were evaluated in phase I or II clinical trials including heavily pretreated EOC patients and generally produced overall response rates (ORRs) of $10-15 \%$, median progression-free survival (PFS) of 2-3 months and median overall survival (OS) of 11-20 months (Table 1). When analysed according 
to biomarker status, PD-L 1 positivity $(2+$ in immunohistochemistry) did not predict objective response in nivolumab trial while objective response to atezolizumab was observed in 2 of 8 patients who had $\geq 5 \%$ PD-L1 expression in immune cells (IC2/3) but not in the patient whose PD-L1 expression was $<5 \%$ (IC0/1) $(15,16)$. In the avelumab study, ORRs in PD-L1 positive and negative cohorts were $11.8 \%$ and $7.9 \%$, respectively, when cut-off for PD-L1 positivity was set at $1 \%(17)$. With a PD-L1 cut-off of $5 \%$, ORRs were $12.5 \%$ and $9.8 \%$, respectively. The KEYNOTE-100 trial was the largest study on ICI monotherapy in OC where PD-L1 expression was measured as combined positive score (CPS), defined as the ratio of $\mathrm{PD}-\mathrm{L} 1$ positive cells (lymphocytes, macrophages and tumor cells) to viable tumor cells (19). Here, ORR to pembrolizumab was reported as $5.0 \%$ for CPS $<1,10.2 \%$ for CPS $\geq 1$ and $17.1 \%$ for CPS $\geq 10$.

Another target of immunotherapeutics is CTLA-4. CTLA-4 is present on T lymphocytes and suppresses $\mathrm{T}$ cell activation by competing for ligands CD80 (B7-1) and CD86 (B7-2) which are expressed by antigen-presenting cells (22). Ipilimumab, a monoclonal antibody against CTLA-4, was administered to 9 advanced OC patients after immunization with granulocyte-macrophage colony-stimulating factor (GM-CSF) and only one patient had a partial response (20). In a phase II trial 40 recurrent platinum-sensitive OC patients were treated with ipilimumab $10 \mathrm{mg} / \mathrm{kg}$ every three weeks for 4 doses and then the same dose every 12 weeks (21). ORR in this study was reported as $10.3 \%$.

Efficacy data from these trials suggest that EOC does not seem to respond well to anti PD-1/PD-L1 or anti-CTLA monotherapy. This can be possibly explained by the fact that only a small proportion of OCs have high tumor mutational burden (TMB), which is defined as the total number of mutations in a tumor specimen and emerged as a novel predictive biomarker for immunotherapy (23). Further limitations in monotherapy trials may be that the majority of patients had received multiple lines of chemotherapy and patient numbers were relatively small in some studies. Although ORR to ICIs was numerically better in PD-L1 positive tumors in some of the trials, the difference could not be statistically proven and optimal predictive cut-off value of PD-L1 is also not clear. It is apparent that better characterization of tumor microenvironment and validated biomarkers are required to select OC patients who may benefit from ICI monotherapy.

\section{Combination of ICls with other agents}

\section{Chemotherapy}

A reasonable approach for increasing tumor immunogenicity and enhancing efficacy of ICIs may be combining them with cytotoxic agents. Chemotherapy can cause release of tumor antigens upon cell death and facilitate phagocytosis by dendritic cells (DCs) which is mediated by damage-associated molecules and augments antigen presentation to $\mathrm{T}$ cells (24). Cell death can also stimulate type 1 interferon (IFN) secretion by tumor cells via toll-like receptor 3 (TLR3) which leads to production of the chemokine CXCL10 (25). Immunomodulatory effects of chemotherapeutics include various processes like promoting cell lysis, impairing $\mathrm{T}_{\text {reg }}$ activity and increasing DC activation (26).

Combination of ICIs with chemotherapy is an active area of research in OC and one of the largest trials based on this design is the phase III JAVELIN Ovarian 200 trial. It included 566 platinum-resistant or platinum-refractory EOC patients who had received up to 3 lines of treatment (27) (Table 2). In this study, addition of avelumab to pegylated liposomal doxorubicin (PLD) did not prolong PFS and OS significantly overall but in PD-L1 positive $(\geq 1 \%$ of tumor cells or $\geq 5 \%$ of immune cells) subgroup an improvement in survival was reported [hazard ratio $(\mathrm{HR})=0.72, \mathrm{P}=0.11$ for PFS and HR $=0.59, \mathrm{P}=0.005$ for OS]. The Avelumab in Previously Untreated Patients With Epithelial Ovarian Cancer (JAVELIN OVARIAN 100, NCT02718417) trial aimed to assess efficacy of avelumab as maintenance therapy following carboplatin/paclitaxel or carboplatin/paclitaxel/ avelumab in 998 treatment-naive advanced EOC patients. The study was discontinued because of futile efficacy in interim analysis.

\section{Anti-angiogenic therapy}

Angiogenesis, defined as formation of new blood vessels, plays a critical role in tumor proliferation and metastasis. It is induced by vascular endothelial growth factor A (VEGF-A) which is secreted by tumor cells and stimulates proliferation of endothelial cells by binding to the receptors VEGFR-1 and VEGFR-2. VEGF-A also 
Table 2 Trials evaluating combinations of immune checkpoint inhibitors with other therapies and dual immune checkpoint blockade in ovarian cancer

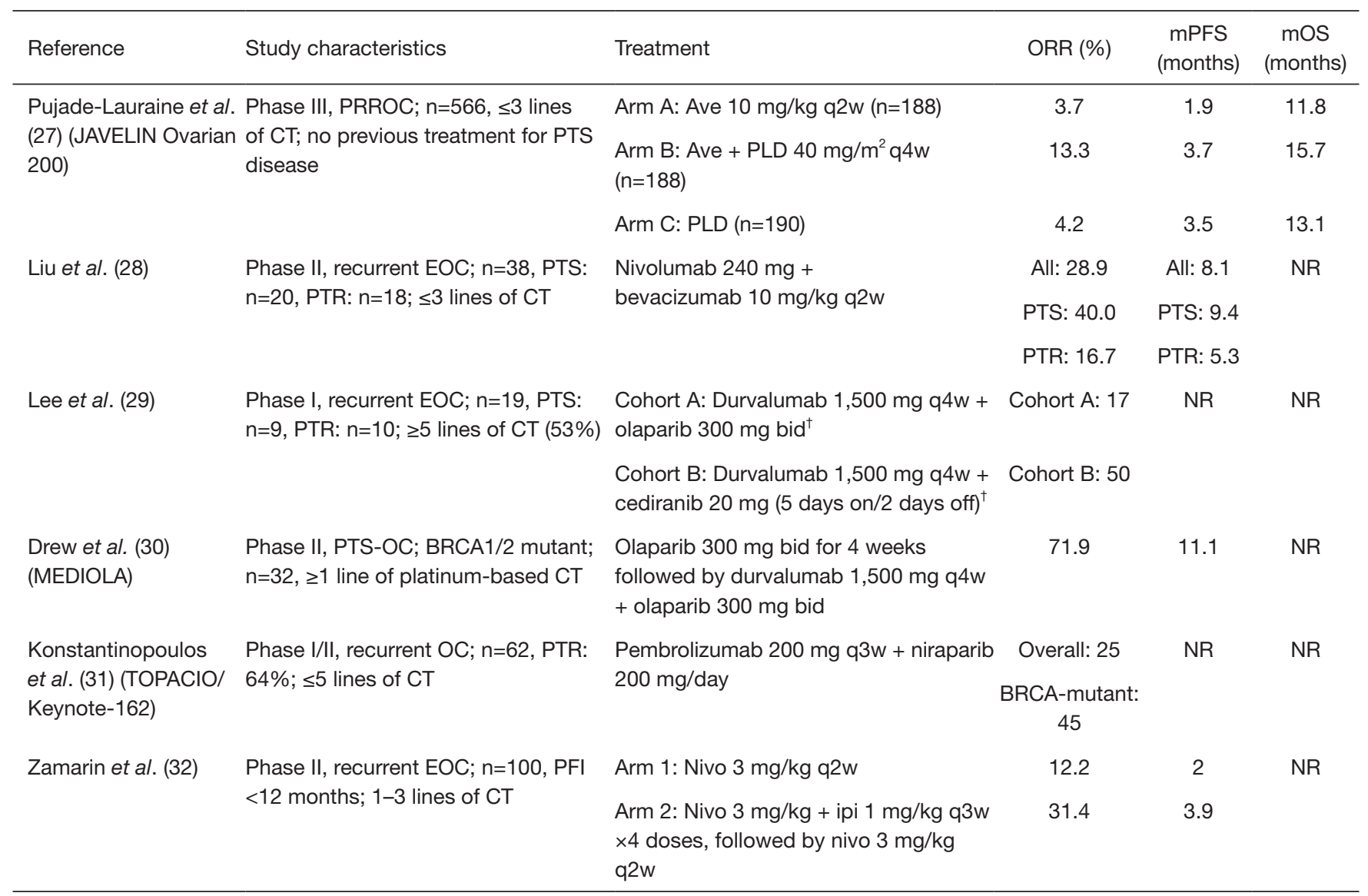

\footnotetext{
${ }^{\dagger}$, final dose levels. Ave, avelumab; Nivo, nivolumab; ipi, ipilimumab CT, chemotherapy; EOC, epithelial ovarian cancer; mOS, median overall survival; mPFS, median progression-free survival; NR, not reported; OC, ovarian cancer; ORR, objective response rate; PFI, platinum-free interval; PLD, pegylated liposomal doxorubicin; PRROC, platinum-resistant and -refractory epithelial ovarian cancer; PTR, platinum-resistant; PTS, platinum-sensitive; PTS-OC, platinum-sensitive ovarian cancer.
}

causes immunosupression by blocking DC maturation and consequently decreasing antigen presentation to $\mathrm{T}$ cells (33). Other mechanisms of angiogenesis-directed immune tolerance are accumulation of immunoregulatory cells $\left(\mathrm{T}_{\text {reg }}\right.$ cells, myeloid-derived suppressor cells) and inhibition of $\mathrm{T}$ cell production and functions (34-37). Therefore, combination with VEGF blockade is another potential method to increase anti-tumor activity of immunotherapy.

Efficacy of nivolumab combined with bevacizumab, an anti-VEGF monoclonal antibody, was investigated in a phase II trial which enrolled 38 relapsed EOC patients (28) (Table 2). Outcomes of this trial revealed clinical activity of the combination, with an ORR of $28.9 \%$ and PFS of 8.1 months, which improved to $40 \%$ and 9.4 months, respectively, in platinum-sensitive subgroup (patients whose disease progressed within 6-12 months after platinum-based chemotherapy). In patients with positive ( $\geq 1 \%)$ PD-L1 expression ORR was $14.3 \%$ whereas patients with negative $(\leq 1 \%)$ PD-L1 expression had an ORR of $45.5 \%$. This result suggests that predictive value of PD-L1 expression for ICI activity in OC is still controversial and future validated studies are necessary in this field.

There are ongoing randomized phase III trials investigating addition of atezolizumab to chemotherapy and/or bevacizumab in different OC settings (Table 3). In IMagyn050 (NCT03038100), previously untreated stage III or IV EOC patients will be randomized in 1:1 design to carboplatin/paclitaxel/bevacizumab plus placebo or atezolizumab arms and co-primary endpoints will be investigator-assessed PFS and OS. The ATALANTE 
Table 3 Ongoing trials investigating immune checkpoint inhibitors combined with other agents, from which no results have been reported so far

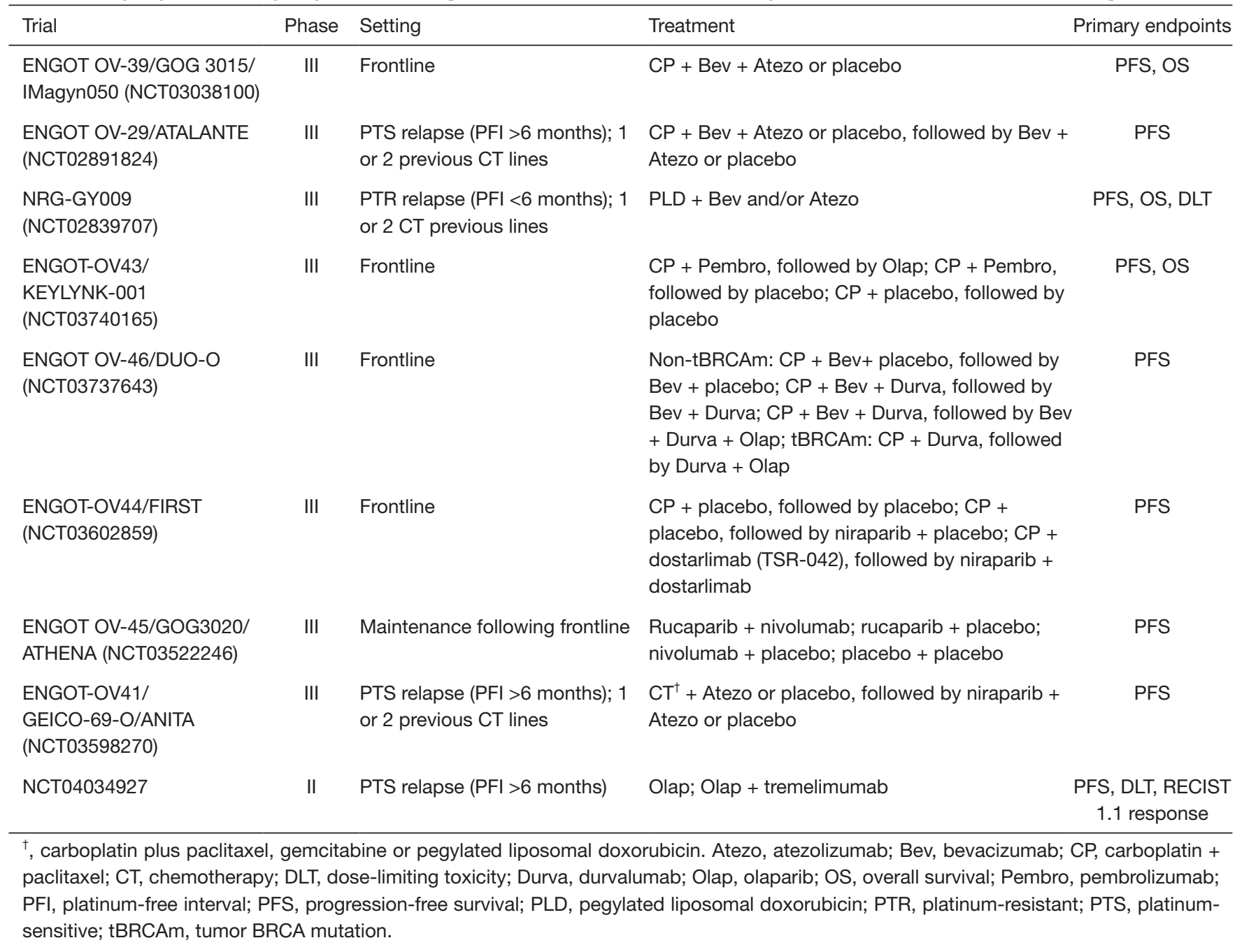

trial (NCT02891824) will assess efficacy of atezolizumab combined with platinum-based chemotherapy/bevacizumab and maintenance bevacizumab in 405 EOC patients who have platinum-sensitive relapse ( $>6$ months). Finally, the NRG-GY009 study (NCT02839707) will evaluate activity of atezolizumab combined with PLD or PLD/bevacizumab in recurrent and platinum-resistant EOC patients who had received one or two previous lines.

\section{PARP inbibitors}

Poly ADP-ribose polymerase (PARP) is a nuclear enzyme which, as a response to DNA damage, synthesizes poly ADP-ribose (PAR) chains to recruit DNA repair proteins. The BRCA1/2 proteins are significant elements of
DNA repair as well and participate in the homologous recombination (HR) pathway to interfere with double strand breaks (38). Clinical activity of PARP inhibitor (PARPi) therapy in various cancers including OC was demonstrated, especially in BRCA-mutant and also nonBRCA mutant HR-deficient cases. US Food and Drug Administration (FDA)-approved PARP inhibitors include olaparib, rucaparib, and niraparib, while veliparib is in the late stage of clinical development (39). Talazoparib inhibits PARP catalytic activity, trapping PARP $1 / 2$ on damaged DNA, and it has been approved by the US FDA for the treatment of metastatic germline BRCA1/2 mutated breast cancers in October 2018 (40). PARPi could potentiate immunotherapeutic activity in many ways. First, they are thought to increase neoantigen burden through DNA 
damage (35). Presence of HR deficiencies like BRCA1/2 mutations cause amplification of TMB and contribute to ICI sensitivity $(41,42)$. Second, PARPi-induced DNA damage could promote recruitment of $T$ cells via the stimulator of interferon genes (STING) pathway and type I IFN (43). Third, upregulation of PD-L1 expression is a possible effect of PARP inhibition (44). Fourth, PARPi can lead to acute inflammation, remodeling of tumor microenvironment and thus enhancement of immune response (45).

A phase I dose-escalation study including heavily pretreated and recurrent EOC patients assessed clinical activity of durvalumab in combination with olaparib, an oral PARPi, or cediranib, an oral VEGF inhibitor (43). ORR in this study was $17 \%$ for olaparib and $50 \%$ for cediranib combination. In three patients with $3+$ TIL infiltration $(>50 \%$ of tumor area) in immunohistochemistry, median duration of response was 14.5 months but PD-L1 positivity $(\geq 5 \%$ ) was not associated with treatment response. Efficacy of durvalumab and olaparib combination was demonstrated in the phase II MEDIOLA trial which enrolled 32 BRCAmutated platinum-sensitive OC patients (30). Updated results from this trial revealed an ORR of $71.9 \%$ and median PFS of 11.1 months while median OS was not reached at that time. TOPACIO/Keynote-162 is a phase I/II study investigating combination of pembrolizumab with niraparib, an oral PARPi, in recurrent OC (31). Interim analysis of the study reported that ORR was $25 \%$ in the overall cohort and $45 \%$ in BRCA-mutated patients. The phase III JAVELIN OVARIAN PARP100 trial was conducted to evaluate chemotherapy plus avelumab combination followed by maintenance avelumab plus talazoparib, an oral PARPi, in previously untreated and advanced OC. This trial was stopped because no benefit was observed with avelumab. The novel combination strategy of olaparib with the CTLA-4-antagonist tremelimumab is under investigation and early ongoing studies are certainly encouraging (46).

Several ongoing studies are exploring ICI/PARPi combinations in various settings of OC and their details are summarized in Table 3.

\section{Other combinations}

ICI monotherapies did not work well in OC so far, as mentioned above, but combination of two ICIs is a promising method aiming to potentiate anti-tumor activity. Preclinical and clinical studies reported that anti-CTLA-4 plus anti-PD-1 blockade enhanced immune response by increasing effector-to-suppressor cell ratios, leading to production of proinflammatory cytokines and modulating peripheral B cell populations $(47,48)$. In a phase II study which enrolled 100 patients with recurrent or persistent OC, nivolumab plus ipilimumab followed by maintenance nivolumab was compared with nivolumab (32) (Table 2). Here, the combination improved response rate significantly (31.4\% vs. $12.2 \%, \mathrm{P}=0.034)$ and reduced the risk of disease progression by $47 \%$. Response was not associated with PDL1 expression in both arms. Efficacy data from this study emphasize the nivolumab/ipilimumab combination as an encouraging option in recurrent $\mathrm{OC}$ but future studies comparing it with standard chemotherapy are required to establish its role.

Radiotherapy is another modality which may cooperate with immunotherapy. Radiation-induced immunosensitization occurs mainly through DNA damage and immunologic cell death as well as upregulation of MHC class I molecules and enhancing presentation of tumor associated antigens (49-51). In a phase I study 73 patients with progressive metastatic solid tumors, including 9 with OC/fallopian tube cancer, received fractionated stereotactic body radiation therapy (SBRT) to 2-4 metastatic sites and then pembrolizumab which was started within 7 days after completion of SBRT (52). The regimen was well tolerated and had clinical activity, with 9 patients having objective response and 21 having stable disease. Nonetheless, it is clear that more specific trials are needed before recommending radioimmunotherapy in OC.

\section{Future directions}

\section{Cancer vaccines}

Tumor-specific immune response could be achieved with vaccination using various antigens. Tumor antigens reported to be present in EOC include New York oesophageal-1 (NY-ESO-1) of the cancer-testis (CT) antigen family and mucin 1 (MUC-1) (53). Epigenetic modulation of CT antigen genes through DNA hypomethylation can increase antigen expression and potential of vaccine efficacy (54). NY-ESO-1 vaccine along with PLD and decitabine, a DNA methyltransferase inhibitor, was administered to recurrent OC patients in a phase I study based on this rationale (55). In this study, disease control rate was $60 \%$ and median duration of response was around 6 months. A randomized phase II trial, which compared a DC vaccine (CVac) targeting MUC-1 with standard-of-care in advanced 
OC, reported a significantly prolonged PFS in patients with complete remission after second-line chemotherapy (>13 vs. 5 months, HR =0.32) (56). With further research on identification of possible targets, tumor vaccines may emerge as a personalized immunologic treatment for OC.

\section{Oncolytic viruses}

An oncolytic virus (OV) infects tumor cells, causes their lysis and then spreads to adjacent tumor cells and metastases; it can also promote indirect cell death by the host immune system (57). OVs can be administered intratumoral, intraperitoneal or intravenously. Several OVs have been studied in OC. Oncolytic herpes simplex virus expressing interleukin-12 (IL-12) was shown to kill murine and human OC cell lines, control OC metastases and improve survival when administered to omentum and peritoneal cavity in a mouse model (58). Other phase I or II trials are investigating adenovirus, measles virus, vaccinia virus and reovirus in OC; anti-tumor activity was reported in some of them. A promising modification of $\mathrm{OV}$ therapy is to combine it with ICIs because OVs can augment immune infiltration in tumors. Combination of intratumoral Newcastle disease virus therapy with antiCTLA-4 blockade has shown a therapeutic effect in animal models, as suggested by rejection of tumors and improvement in survival (59). Despite these developments, further clinical research is needed to clarify the role of OVs in $\mathrm{OC}$ and before approving them for treatment.

\section{Cellular therapy}

Adoptive cell therapy (ACT) is one of the promising immunologic prospects in oncology. It uses autologous or allogeneic lymphocytes isolated from tumor or peripheral blood through leukopheresis. These are cultured and activated ex vivo and then re-infused to the patient with recombinant interleukin-2 (rIL-2) after lymphodepleting chemotherapy (60). Early data regarding ACT in OC came from a phase I trial of Fujita $e t$ al. who treated 13 EOC patients with adoptive TIL therapy following surgery and cisplatin-based chemotherapy (61). They observed a 3 -year survival of $100 \%$ in these patients versus $67.5 \%$ in the control group which did not receive TILs. In a second phase I trial, autologous vaccine-primed CD3/CD28-costimulated $\mathrm{T}$ lymphocytes were transferred to recurrent EOC patients following DC-based autologous vaccination and lymphodepletion (62). Treatment was well tolerated and anti-tumor response along with clinical benefit was reported.

A major drawback of ACT is that tumor-specific lymphocytes are difficult to obtain. To overcome this, $\mathrm{T}$ cells can be genetically modified by T-cell receptors (TCRs) and chimeric antigen receptors (CARs). Introduction of TCRs targeting the NY-ESO-1 antigen, which is expressed in OC, can enable harvesting tumor-specific $\mathrm{T}$ cells in large numbers (63). On the other hand, $T$ cells can be engineered by CARs to recognize tumor antigens in an MHC-independent manner (64). CARs relevant to OC have been investigated so far and include folate receptor- $\alpha$ (FR- $\alpha$ ), human epidermal growth factor receptor 2 (HER-2) and mesothelin (65-67). A phase I trial of Tanyi et al. demonstrated detectable $\mathrm{T}$ cells in peripheral blood of OC patients with mesothelin-expressing tumors after infusion of autologous $\mathrm{T}$ cells transduced to express a CAR directed against mesothelin (68). Ongoing clinical trials are evaluating TCR- or CAR-redirected T cells against NYESO-1 and mesothelin in OC.

\section{Conclusions}

Preclinical studies revealed immunogenicity of OC and activation of anti-cancer immunity is hence a reasonable therapeutic maneuver for the disease which commonly recurs. Early efforts on this approach evaluated cytokine treatment in OC but failed to present convincing phase III data. On the other hand, ICIs have emerged as significant immunostimulatory agents with increasing use in oncology and immunologic properties of OC provides the basis for introducing them to disease management. However, ICIs as monotherapy brought only modest efficacy when assessed in pretreated OC patients, necessitating additional methods for potentialization. Following this, several strategies aimed to sensitize OC to immunotherapy by combining it with chemotherapy, anti-angiogenetics, PARPi, radiotherapy and by dual immune checkpoint blockade. There are numerous ongoing trials investigating these approaches and some results reported so far indicate better outcomes than ICI monotherapy, especially in terms of response rate. A major concern hereby would be definition of optimal predictive biomarkers to better identify candidates for ICI treatment. Finally, current translational research focuses on other promising immunologic therapies including cancer vaccines, virotherapy and cellular therapy which showed clinical activity in some studies and may emerge as treatment options for $\mathrm{OC}$ in the future. 


\section{Acknowledgments}

Funding: None.

\section{Footnote}

Provenance and Peer Review: This article was commissioned by the Guest Editors (Stergios Boussios and Nicholas Pavlidis) for the series "Ovarian Cancer: State of the Art and Perspectives of Clinical Research" published in Annals of Translational Medicine. The article was sent for external peer review organized by the Guest Editors and the editorial office.

Peer Review File: Available at http://dx.doi.org/10.21037/ atm-20-4499

Conflicts of Interest: All authors have completed the ICMJE uniform disclosure form (available at http://dx.doi. org/10.21037/atm-20-4499). The series "Ovarian Cancer: State of the Art and Perspectives of Clinical Research" was commissioned by the editorial office without any funding or sponsorship. SB served as the unpaid Guest Editor of the series and serves as an unpaid editorial board member of Annals of Translational Medicine from Nov 2019 to Oct 2021. The other authors have no other conflicts of interest to declare.

Ethical Statement: The authors are accountable for all aspects of the work in ensuring that questions related to the accuracy or integrity of any part of the work are appropriately investigated and resolved.

Open Access Statement: This is an Open Access article distributed in accordance with the Creative Commons Attribution-NonCommercial-NoDerivs 4.0 International License (CC BY-NC-ND 4.0), which permits the noncommercial replication and distribution of the article with the strict proviso that no changes or edits are made and the original work is properly cited (including links to both the formal publication through the relevant DOI and the license). See: https://creativecommons.org/licenses/by-nc-nd/4.0/.

\section{References}

1. Howlader NNA, Krapcho M, Garshell J, et al. SEER Cancer Statistics Review. National Cancer Institute; Bethesda, MD: pp. 1975-2012. based on November 2014
SEER data submission, posted to the SEER web site, April 2015 ed.

2. Torre LA, Bray F, Siegel RL, et al. Global cancer statistics, 2012. CA Cancer J Clin 2015;65:87.

3. Zhang L, Conejo-Garcia JR, Katsaros D, et al. Intratumoral T cells, recurrence, and survival in epithelial ovarian cancer. N Engl J Med 2003;348:203-13.

4. Schlienger K, Chu CS, Woo EY, et al. TRANCEand CD40 ligand-matured dendritic cells reveal MHC class I-restricted $\mathrm{T}$ cells specific for autologous tumor in late-stage ovarian cancer patients. Clin Cancer Res 2003;9:1517-27.

5. Pujade-Lauraine E, Guastalla JP, Colombo N, et al. Intraperitoneal recombinant interferon gamma in ovarian cancer patients with residual disease at second-look laparotomy. J Clin Oncol 1996;14:343-50.

6. Alberts DS, Marth C, Alvarez RD, et al. Randomized phase 3 trial of interferon gamma- $1 \mathrm{~b}$ plus standard carboplatin/paclitaxel versus carboplatin/paclitaxel alone for first-line treatment of advanced ovarian and primary peritoneal carcinomas: results from a prospectively designed analysis of progression-free survival. Gynecol Oncol 2008;109:174-81.

7. Recchia F, Di Orio F, Candeloro G, et al. Maintenance immunotherapy in recurrent ovarian cancer: long term follow-up of a phase II study. Gynecol Oncol 2010;116:202-7.

8. Vlad AM, Budiu RA, Lenzner DE, et al. A phase II trial of intraperitoneal interleukin-2 in patients with platinumresistant or platinum-refractory ovarian cancer. Cancer Immunol Immunother 2010;59:293-301.

9. Simpkins F, Flores A, Chu C, et al. Chemoimmunotherapy using pegylated liposomal doxorubicin and interleukin-18 in recurrent ovarian cancer: a phase I dose-escalation study. Cancer Immunol Res 2013;1:168-78.

10. Hamanishi J, Mandai M, Iwasaki M, et al. Programmed cell death 1 ligand 1 and tumor-infiltrating $\mathrm{CD} 8+\mathrm{T}$ lymphocytes are prognostic factors of human ovarian cancer. Proc Natl Acad Sci USA 2007; 104:3360-5.

11. Maine CJ, Aziz NH, Chatterjee J, et al. Programmed death ligand-1 over-expression correlates with malignancy and contributes to immune regulation in ovarian cancer. Cancer Immunol Immunother 2014;63:215-24.

12. Cheng B, Liang H, Jiang T, et al. Characterization of PD$\mathrm{L} 1$ expression and its prognostic value in patients with ovarian cancer. Transl Cancer Res 2018;7:1271-81.

13. Goode EL, Block MS, Kalli KR, et al. Dose-response relationship of CD8+ tumor infiltrating lymphocytes and 
survival time in high-grade serous ovarian cancer. JAMA Oncol 2017;3:e173290.

14. Francisco LM, Salinas VH, Brown KE, et al. PD-L1 regulates the development, maintenance, and function of induced regulatory T cells. J Exp Med 2009;206:3015-29.

15. Hamanishi J, Mandai M, Ikeda T, et al. Safety and antitumor activity of anti-PD-1 antibody, nivolumab, in patients with platinum-resistant ovarian cancer. J Clin Oncol 2015;33:4015-22.

16. Liu JF, Gordon M, Veneris J, et al. Safety, clinical activity and biomarker assessments of atezolizumab from a phase I study in advanced/recurrent ovarian and uterine cancers. Gynecol Oncol 2019;154:314-22.

17. Disis ML, Taylor MH, Kelly K, et al. Efficacy and safety of avelumab for patients with recurrent or refractory ovarian cancer: phase $1 \mathrm{~b}$ results from the JAVELIN solid tumor trial. JAMA Oncol 2019;5:393-401.

18. Varga A, Piha-Paul S, Ott PA, et al. Pembrolizumab in patients with programmed death ligand 1-positive advanced ovarian cancer: analysis of KEYNOTE-028. Gynecol Oncol 2019;152:243-50.

19. Matulonis UA, Shapira-Frommer R, Santin AD, et al. Antitumor activity and safety of pembrolizumab in patients with advanced recurrent ovarian cancer: results from the Phase 2 KEYNOTE-100 study. Ann Oncol 2019;30:1080-7.

20. Hodi FS, Butler M, Oble DA, et al. Immunologic and clinical effects of antibody blockade of cytotoxic $T$ lymphocyte-associated antigen 4 in previously vaccinated cancer patients. Proc Natl Acad Sci USA 2008;105:3005-10.

21. NCT01611558: Phase II Study of Ipilimumab Monotherapy in Recurrent Platinum-sensitive Ovarian Cancer - Study Results. Available online: https:// clinicaltrials.gov/ct2/show/results/NCT01611558. Accessed 13 March 2020.

22. Wei SC, Duffy CR, Allison JP. Fundamental mechanisms of immune checkpoint blockade therapy. Cancer Discov 2018; 8: 1069-86.

23. Chalmers ZR, Connelly CF, Fabrizio D, et al. Analysis of 100,000 human cancer genomes reveals the landscape of tumor mutational burden. Genome Med 2017;9:34.

24. Kepp O, Galluzzi L, Martins I, et al. Molecular determinants of immunogenic cell death elicited by anticancer chemotherapy. Cancer Metastasis Rev 2011;30:61-9.

25. Sistigu A, Yamazaki T, Vacchelli E, et al. Cancer cell-autonomous contribution of type I interferon signaling to the efficacy of chemotherapy. Nature Med 2014;20:1301-9.

26. Chen G, Emens LA. Chemoimmunotherapy: reengineering tumor immunity. Cancer Immunol Immunother 2013;62:203-16.

27. Pujade-Lauraine E, Fujiwarab K, Ledermann JA, et al. Avelumab alone or in combination with pegylated liposomal doxorubicin versus pegylated liposomal doxorubicin alone in platinum-resistant or refractory epithelial ovarian cancer: Primary and biomarker analysis of the phase III JAVELIN Ovarian 200 trial. Gynecol Oncol 2019;154:21-2.

28. Liu JF, Herold C, Gray KP, et al. Assessment of combined nivolumab and bevacizumab in relapsed ovarian cancer: a phase 2 clinical trial. JAMA Oncol 2019;5:1731-8.

29. Lee JM, Cimino-Mathews A, Peer CJ, et al. Safety and clinical activity of the programmed death-ligand 1 inhibitor durvalumab in combination with poly (ADPribose) polymerase inhibitor olaparib or vascular endothelial growth factor receptor 1-3 inhibitor cediranib in women's cancers: a dose-escalation, phase I study. J Clin Oncol 2017;35:2193-202.

30. Drew Y, Kaufman B, Banerjee S, et al. Phase II study of olaparib 1 durvalumab (MEDIOLA): updated results in germline BRCA-mutated platinum-sensitive relapsed (PSR) ovarian cancer (OC). Ann Oncol 2019;30:v485-486.

31. Konstantinopoulos PA, Waggoner SE, Vidal GA, et al. TOPACIO/Keynote-162 (NCT02657889): a phase 1/2 study of niraparib + pembrolizumab in patients (pts) with advanced triple-negative breast cancer or recurrent ovarian cancer (ROC) - results from ROC cohort. J Clin Oncol 2018;36:abstr 106.

32. Zamarin D, Burger RA, Sill MW, et al. Randomized Phase II Trial of Nivolumab Versus Nivolumab and Ipilimumab for Recurrent or Persistent Ovarian Cancer: An NRG Oncology Study. J Clin Oncol 2020;38:1814-23.

33. Gabrilovich DI, Chen HL, Girgis KR, et al. Production of vascular endothelial growth factor by human tumors inhibits the functional maturation of dendritic cells. Nat Med 1996;2:1096-103.

34. Terme M, Pernot S, Marcheteau E, et al. VEGFAVEGFR pathway blockade inhibits tumor-induced regulatory T-cell proliferation in colorectal cancer. Cancer Res 2013;73:539-49.

35. Gabrilovich D, Ishida T, Oyama T. Vascular endothelial growth factor inhibits the development of dendritic cells and dramatically affects the differentiation of multiple hematopoietic lineages in vivo. Blood 1998;92:4150-66. 
36. Ohm JE, Gabrilovich DI, Sempowski GD, et al. VEGF inhibits T-cell development and may contribute to tumorinduced immunesuppression. Blood 2003;101:4878-86.

37. Ziogas AC, Gavalas NG, Tsiatas M, et al. VEGF directly suppresses activation of $\mathrm{T}$ cells from ovarian cancer patients and healthy individuals via VEGF receptor Type 2. Int J Cancer 2012;130:857-64.

38. Boussios S, Karathanasi A, Cooke D, et al. PARP Inhibitors in Ovarian Cancer: The Route to "Ithaca". Diagnostics (Basel) 2019;9:55.

39. Boussios S, Karihtala P, Moschetta M, et al. Veliparib in ovarian cancer: a new synthetically lethal therapeutic approach. Invest New Drugs 2020;38:181-93.

40. Boussios S, Abson C, Moschetta M, et al. Poly (ADPRibose) Polymerase Inhibitors: Talazoparib in Ovarian Cancer and Beyond. Drugs R D 2020;20:55-73.

41. Li A, Yi M, Qin S, et al. Prospects for combining immune checkpoint blockade with PARP inhibition. J Hematol Oncol 2019;12:98.

42. Pilié PG, Gay CM, Byers LA, et al. PARP inhibitors: extending benefit beyond BRCA-mutant cancers. Clin Cancer Res 2019;25:3759-71.

43. Pantelidou C, Sonzogni O, De Oliveira TM, et al. PARP inhibitor efficacy depends on CD8+ T cell recruitment via intratumoral STING pathway activation in BRCAdeficient models of triple negative breast cancer. Cancer Discov 2019;9:722-37.

44. Jiao S, Xia W, Yamaguchi H, et al. PARP inhibitor upregulates PD-L1 expression and enhances cancerassociated immunosuppression. Clin Cancer Res 2017;23:3711-20.

45. Chabanon RM, Muirhead G, Krastev DB, et al. PARP inhibition enhances tumor cell-intrinsic immunity in ERCC1-deficient non-small cell lung cancer. J Clin Invest 2019;129:1211-28.

46. Boussios S, Karihtala P, Moschetta M, et al. Combined Strategies with Poly (ADP-Ribose) Polymerase (PARP) Inhibitors for the Treatment of Ovarian Cancer: A Literature Review. Diagnostics (Basel) 2019;9:87.

47. Curran MA, Montalvo W, Yagita H, et al. PD-1 and CTLA-4 combination blockade expands infiltrating $\mathrm{T}$ cells and reduces regulatory $\mathrm{T}$ and myeloid cells within B16 melanoma tumors. Proc Natl Acad Sci USA 2010;107:4275-80.

48. Das R, Bar N, Ferreira M, et al. Early B cell changes predict autoimmunity following combination immune checkpoint blockade. J Clin Invest 2018;128:715-20.

49. Joiner MC, Marples B, Lambin P, et al. Low-dose hypersensitivity: current status and possible mechanisms. Int J Radiat Oncol Biol Phys 2001;49:379-89.

50. Herrera FG, Bourhis J, Coukos G. Radiotherapy combination opportunities leveraging immunity for the next oncology practice. CA Cancer J Clin 2017;67:65-85.

51. Reits EA, Hodge JW, Herberts CA, et al. Radiation modulates the peptide repertoire, enhances $\mathrm{MHC}$ class I expression, and induces successful antitumor immunotherapy. J Exp Med 2006;203:1259-71.

52. Luke JJ, Lemons JM, Karrison TG, et al. Safety and clinical activity of pembrolizumab and multisite stereotactic body radiotherapy in patients with advanced solid tumors. J Clin Oncol 2018;36:1611-8.

53. Odunsi K, Qian F, Matsuzaki J, et al. Vaccination with an NY-ESO-1 peptide of HLA class I/II specificities induces integrated humoral and $\mathrm{T}$ cell responses in ovarian cancer. Proc Natl Acad Sci USA 2007;104:12837-42.

54. Karpf AR. A potential role for epigenetic modulatory drugs in the enhancement of cancer/germ-line antigen vaccine efficacy. Epigenetics 2006;1:116-20.

55. Odunsi K, Matsuzaki J, James SR, et al. Epigenetic potentiation of NY-ESO-1 vaccine therapy in human ovarian cancer. Cancer Immunol Res 2014;2:37-49.

56. Gray HJ, Benigno B, Berek J, et al. Progression-free and overall survival in ovarian cancer patients treated with CVac, a mucin 1 dendritic cell therapy in a randomized phase 2 trial. J Immunother Cancer 2016;4:34.

57. Russell SJ, Peng, KW. Oncolytic virotherapy: a contest between apples and oranges. Mol Ther 2017;25:1107-16.

58. Thomas ED, Meza-Perez S, Bevis KS, et al. IL-12 expressing oncolytic herpes simplex virus promotes antitumor activity and immunologic control of metastatic ovarian cancer in mice. J Ovarian Res 2016;9:70.

59. Zamarin D, Holmgaard RB, Subudhi SK, et al. Localized oncolytic virotherapy overcomes systemic tumor resistance to immune checkpoint blockade immunotherapy. Sci Transl Med 2014;6:226ra32.

60. Rosenberg SA, Restifo NP, Yang JC, et al. Adoptive cell transfer: a clinical path to effective cancer immunotherapy. Nat Rev Cancer 2008;8:299-308.

61. Fujita K, Ikarashi H, Takakuwa K, et al. Prolonged disease-free period in patients with advanced epithelial ovarian cancer after adoptive transfer of tumor-infiltrating lymphocytes. Clin Cancer Res 1995;1:501-7.

62. Kandalaft LE, Powell DJ, Chiang CL, et al. Autologous lysate-pulsed dendritic cell vaccination followed by adoptive transfer of vaccine primed ex vivo co-stimulated $\mathrm{T}$ cells in recurrent ovarian cancer. Oncoimmunology 
2013;2:e22664-9.

63. Rosati SF, Parkhurst MR, Hong Y, et al. A novel murine T-cell receptor targeting NY-ESO-1. J Immunother 2014;37:135-46.

64. Sadelain $M$, Brentjens R, Rivière I. The promise and potential pitfalls of chimeric antigen receptors. Curr Opin Immunol 2009;21:215-23.

65. Kandalaft LE, Powell DJ, Coukos G. A phase I clinical trial of adoptive transfer of folate receptor-alpha redirected autologous T cells for recurrent ovarian cancer. J Transl Med 2012;10:157.

66. Lanitis E, Dangaj D, Hagemann IS, et al. Primary human ovarian epithelial cancer cells broadly express

Cite this article as: Demircan NC, Boussios S, Tasci T, Öztürk MA. Current and future immunotherapy approaches in ovarian cancer. Ann Transl Med 2020;8(24):1714. doi: 10.21037/ atm-20-4499
HER2 at immunologically-detectable levels. PLoS ONE 2012;7:e49829.

67. Chang K, Pastan I. Molecular cloning of mesothelin, a differentiation antigen present on mesothelium, mesotheliomas, and ovarian cancers. Proc Natl Acad Sci USA 1996;93:136-40.

68. Tanyi JL, Hass AR, Beatty GL et al. Safety and feasibility of chimeric antigen receptor modified $\mathrm{T}$ cells directed against mesothelin (CART-meso) in patients with mesothelin expressing cancers. Presented at: 2015 American Association for Cancer Research Annual Meeting; April 18-22, 2015; Philadelphia, PA. Abstract CT105. 